\title{
RETOS Y COMPROMISOS
}

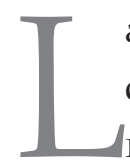

a edición 12 de la Revista PALOBRA "Palabra que Obra", comprometida con el proceso de internacionalización e integración a sistemas de información y a la espera de su revaloración en la Red de Revistas Científicas de América Latina, el Caribe, España y Portugal (Redalyc), dio pasos significativos para atender los requerimientos de indexación de revistas colombianas de ciencia, tecnología e innovación, tanto en la Base Bibliográfica Nacional -BBN- y el Índice Bibliográfico Publindex -IBN-, mostrando el interés pleno por mantener la calidad editorial.

Igualmente, en esta edición ampliamos el cumplimiento de la valoración de condiciones y exigencias representadas en el perfil de los miembros del comité editorial y las titulaciones de los pares externos, para seguir atendiendo los indicadores internacionales como es el de ampliar su número de articulistas, trascendiendo los espacios institucionales y locales.

La Revista PALOBRA "Palabra que Obra" participó y firmó convenio marco con la Revista Historia de la Educación Latinoamericana, comprometiéndose con los pares editoriales de doce revistas del continente para fortalecer lazos de colaboración en la realización de asuntos editoriales conjuntos y para la difusión y promoción de los mismos.

La comunidad académica y científica de las Ciencias Sociales, Humanas y Educación, en los ámbitos nacional e internacional se hizo presente en la edición 12 de la Revista PALOBRA "Palabra que Obra", compartiendo diversos problemas, enfoques y paradigmas investigativos que sustentan cada uno de los temas de los artículos recibidos, evaluados y puestos bajo el criterio de los pares de Colciencias, quienes asumieron los procesos con rigurosidad.

Los profesores Ricardo Pérez Montfort y Gabriela Pulido Llano, pertenecientes al Centro de Investigaciones y Estudios Superiores en Antropología Social (CIESAS) y el Instituto Nacional de Antropología e Historia, quienes se vinculan por primera vez a esta revista, presentan en esta edición su artículo "CULTURA CUBANA Y MEDIOS DE COMUNICACIÓN EN MÉXICO, 1920-1950", en el que analizan la influencia de la cultura popular cubana en la cultura popular mexicana, a través de los medios de comunicación, mostrando como 
las características de los estereotipos culturales identificados con lo cubano, trajeron como resultado un producto de exportación que los medios mexicanos aprovecharon de maneras muy creativas, particularmente en la música y el cine producidos en los años treinta y cuarenta, así como en los teatros, salones de baile y cabarets. Sostienen Pérez y Pulido que detrás de todas estas manifestaciones culturales "hubo una nutrida migración de artistas cubanos que contribuyeron a la manufactura de una vertiente de la cultura popular mexicana asociada a lo tropical".

El artículo "MADRES Y PADRES EN VENEZUELA. UNA TRADICIÓN QUE SE SOSTIENE ENTRE CADENAS Y REDES MIGRATORIAS. CASO CARTAGENA Y BA$R R A N Q U I L L A$ ", de Pilar Morad, Gloria Bonilla y Mercedes Rodríguez López, es producto de la investigación inter-universitaria e interdisciplinaria "Cambios y conflictos de los grupos familiares frente a la migración internacional", que se desarrolló con cofinanciación de Colciencias y la coparticipación de cinco universidades colombianas: Universidad Nacional, Universidad de Antioquia, Universidad del Valle, Universidad de Caldas, y Universidad de Cartagena.

El análisis se centra en la reflexión sobre las redes familiares y las cadenas migratorias que soportan la migración de padres y madres, que se han generado históricamente hacia Venezuela desde algunas ciudades de la costa Caribe colombiana, como Cartagena y Barranquilla, dada su proximidad fronteriza.

Soportan su estudio en el análisis de los relatos de cuidadoras/es e hijas/os que se quedan en las ciudades de origen, para comprender la dinámica del proceso migratorio hacia este país, los factores del contexto que la impulsan y el papel de las redes que reproducen el tránsito permanente de padres y madres en busca de mejores oportunidades para sus familias.

Javier Hernández y Liliana Núñez Martínez, en su artículo "VECINOS $Y$ CIUDADANOS: ¿PARA MITIGAR LA ADVERSIDAD O PARA ESTAR EN EL PODER DIRECTAMENTE?", intentan mostrar desde testimonios de líderes de la ciudad de Cartagena, cómo el ejercicio de la acción participativa desde algunas agencias institucionalizadas cívico-políticas se inserta en las lógicas y escenarios de los procesos de urbanización, en particular los de poblamiento y consolidación barrial, estableciéndose entre aquél y éstos unas modulaciones específicas, empíricamente determinables. Argumentan que las prácticas de ciudadanía consideradas en su estudio no se despliegan de modo absoluto en un territorio neutral, la ciudad, sino que son condicionadas por ésta y, al 
tiempo, la constituyen en lo que es: concurrencia competitiva y desigual por el derecho a la ciudad por parte de agencias y agentes jerarquizados en un medio urbano fragmentado y segregado.

El artículo es un avance del proyecto de investigación en marcha, financiado por la Universidad de Cartagena, "La construcción de ciudad como pacto colectivo: estrategias tradicionales y emergentes de participación y representación cívico-políticas en Cartagena de Indias."

En esta edición también se evidencia la presencia de estudiantes de la Facultad de Ciencias Sociales y Educación de la Universidad de Cartagena, miembros de semilleros de investigación quienes oxigenan estas páginas de la Revista Palobra, y aseguran con su presencia los relevos generacionales a que aspiramos los profesores e investigadores. El artículo "TERRITORIOS PROHIBIDOS", LAS MUJERES EN EL CAMINO HACIA LA VIDA POLÍTICA, CARTAGENA DURANTE LA PRIMERA MITAD DEL SIGLO XX", de Raúl Cera Ochoa, Nathaly Ortega Polanco y Carlos Castrillón, corresponde a un documento de trabajo presentado para participar en la IV convocatoria interna para proyectos de investigación en el año 2011 por el grupo de investigación Estudios de Familias. Masculinidades y Feminidades, de la citada facultad.

En este artículo se presenta un conjunto de hechos que durante las primeras décadas del siglo XX en Cartagena promueven la inclusión y participación de las mujeres a la vida política de esta ciudad. Se trata de analizar el camino que tuvieron que recorrer para lograr el reconocimiento pleno de los derechos políticos y trascender la condición que la sociedad les había atribuido: las labores domésticas; las costumbres puritanas, la moral cristiana y los estereotipos de género patriarcales, lo que impedía de hecho y formalmente su participación en asuntos públicos.

Respondió también a nuestra convocatoria, la profesora Mg. Gabriela Beatriz Rotondi, de Córdova, Argentina, quien socializa el trabajo de investigación "AGREMLACIÓN Y ACCIÓN JUVENIL EN ESPACIOS EDUCATIVOS", referido al estudio de los derechos gremiales de los estudiantes secundarios en las escuelas públicas. En el artículo, Rotondi muestra cómo las dificultades organizativas se encuentran relacionadas con las trayectorias estudiantiles en las instancias organizativas como los centros de estudiantes, las estructuras de poder institucional y normativo instaladas en las escuelas, y las particulares condiciones de los escenarios escolares. El juego de los diversos sujetos presentes en la

PalObra №. 12. Agosto de 2010 - Julio de 2011 
institución se complejiza por la dinámica y ejercicio del poder en la escena institucional escolar. Al respecto surgen las siguientes preguntas: ¿qué parámetros organizativos se plantean respecto de la participación ciudadana de los jóvenes estudiantes?, ¿qué viabilidad plantean los gérmenes organizativos de estudiantes secundarios en los formatos actuales para la agremiación juvenil?

Los derechos ciudadanos, sean civiles, políticos, sociales, gremiales o reproductivos, permanecen condicionados en términos estructurales por la lógicas de organización social tanto como por los espacios sociales e institucionales, incluida la escuela.

Por su parte, Nicolás Simarra Torres, profesor investigador nos presenta el ensayo "COLOMBIA Y SUS COMPROMISOS CON LA PRIMERA INFANCLA", en el cual se abordan los argumentos por los cuales es importante invertir en este período de la vida del ser humano, destacando programas relevantes en este ámbito y la incidencia de organismos internacionales en el tema. También se aborda la trayectoria de Colombia en la atención a la primera infancia, discriminando varios escenarios: los primeros, los recientes y el momento actual, lo que aporta una visión sobre los avances, pero también los grandes retos a que se enfrenta el país en este tema.

Laura Taylor profesora del Departamento de sicología del instituto internacional de estudios de paz de la Universidad de Notre Dame, socializa los resultados de la investigación, "RELACIONES ENTRE LA VIOLENCIA, SALUD MENTAL, PARTICIPACIÓN CIUDADANA Y ACTITUDES HACIA LA JUSTICIA TRANSICIONAL EN LA COSTA CARIBE COLOMBIANA", que fue financiada por Kellogg Institute for International Studies, Kroc Institute for International Peace Studies y Institute for Scholarship in the Liberal Arts de la University of Notre Dame, con la participación de equipos de investigadores de la universidades del Caribe (del Sinú, de Córdoba y CECAR en Sincelejo), así como también, la comisión ciudadana de Reconciliación en el Caribe CCRC.

El artículo explora por un lado, que la violencia política tiene el potencial de amenazar el bienestar de los individuos y romper el tejido social y, por otro lado, que los individuos y comunidades son resilientes frente a ella. De este modo, plantea la siguiente pregunta de investigación: ¿Cómo las experiencias de violencia política afectan a las personas a nivel personal?, así como también, explora las relaciones entre violencia, salud mental, participación ciudadana y actitudes hacia la justicia transicional y democracia dentro un contexto de 
conflicto armado prolongado. Una contribución teórica de este proyecto es la integración de dos marcos teóricos de psicología y estudios de paz. Los resultados sugieren la necesidad de desarrollar intervenciones de salud mental y construcción de paz más sensibles al contexto y menos centralizadas.

El articulo "ORDEN, PODER Y CONTRABANDO EN EL CARIBE DURANTE EL MEDIO SIGLO ANTES DE LA INDEPENDENCI $A$ ", escrito por la profesora Ruth Gutiérrez Meza de la Facultad de Ciencias Humanas de la Universidad de Cartagena, recrea como el Caribe se ha caracterizado históricamente por su diversidad, heterogeneidad e importancia geográfica para el comercio y argumenta así mismo, que durante el medio siglo que precedió a la independencia este territorio adquirió una significativa y estratégica importancia a nivel mercantil que le permitió figurar como el centro de disputas entre las metrópolis europeas y cómo estas poblaciones desarrollaron paralelamente prácticas sociales y estrategias económicas como el contrabando, que en su vida diaria les permitieron beneficiarse del contexto político y económico que les rodeaba.

El profesor de la Universidad de Chile, Lorenzo Agar Corbinos, nos ilustra sobre un tema relevante y cada vez más vigente en el presente siglo, a través de su artículo "MIGRACIONES POSMODERNAS EN CHILE: REFLEXIONES SOBRE COHESIÓN SOCIAL Y PLURALIDAD CULTURAL". Como experto en el tema, asume que las naciones receptoras de inmigrantes deben velar por generar equitativas condiciones de vida para el conjunto de la población. En este artículo se ejemplifican las nuevas migraciones con el caso chileno pues se evidencia, un importante flujo inmigratorio en las dos últimas décadas; lo cual plantea los problemas de la inserción de los grupos culturales foráneos en un ambiente local, con una tendencia histórica a la representación social de un homogéneo más, que transita con fuerza hacia ambientes de pluralidad cultural, en un contexto de globalización y posmodernidad. Es esta tensión social la que ha expresado en este texto como una forma de motivar el pensamiento reflexivo en torno a los tránsitos hacia una sociedad más abierta ideológicamente, diversa culturalmente e imperativamente más participativa y dialogante.

"LA GESTIÓN DE LA IMAGEN SUBJETTVA Y OBJETIVA DE LA EMPRESA EN LOS NUEVOS CANALES COMERCIALES” se titula el artículo presentado por el profesor David López Jiménez, doctor en Derecho de la Universidad de Huelva, España, quien asume que la reputación corporativa es un valor 
difícil de crear, pero muy fácil de perder. Tal aspecto se proyecta tanto en el mundo tradicional como en el escenario virtual. En la formación de la imagen empresarial influyen numerosos factores, si bien, en la actualidad, los instrumentos sociales presentes en internet amplifican los efectos, positivos o negativos, respecto a la misma; una vez cuantificada, podrá monitorizarse para realizar las medidas pertinentes.

Ricardo Chica, candidato a doctor en Ciencias de la Educación, profesor de la Universidad de Cartagena, socializa y dinamiza el estudio "MEMORLA RADIAL SOLLE: DINÁMICAS URBANAS, MÚSICA MUNDO E IDENTIDAD JUVENIL ENLOS SECTORES POPULARES DE CARTAGENA (1975 - 1985)". Este estudio centra su atención en los sentidos que se produjeron socialmente en el consumo de la llamada música "solle" en Cartagena durante los años setenta y ochenta , y pretende comprenderlos. Dichos sentidos se inscribieron en prácticas culturales que partieron de la oferta identitaria hecha por la producción discográfica venida de Estados Unidos y Europa y por los medios de comunicación, en especial, la radio local y que dieron lugar a la emergencia de una comunidad urbana de jóvenes que imaginaron la ciudad de una cierta forma y su relación con la cultura-mundo. Esta comunidad fue conocida entonces como "solles", una palabra que es contracción de sollado o arrebatado. Sentidos que, a su vez, se inscriben en un territorio virtual y, en esa lógica, superan las fronteras nacionales para conectarse con coordenadas planetarias de identidad juvenil mediadas por la música. Para pensar el fenómeno se adopta un doble enfoque: la sociología de la cultura y la historia social de los medios e interrogar las condiciones en que se dio la relación identidad juvenil - cultura mundo - dinámicas urbanas. Para ello se seleccionaron informantes clave para llevar a cabo historias de vida, lo que se apoyó con labor de archivo de prensa y análisis del contexto mediante fotografías, entre otras actividades.

En el mismo sentido, el profesor Ricardo Pérez Montfort, del Centro de Investigaciones y Estudios Superiores en Antropología Social (Ciesas), la Dirección de Estudios de Historia y el Instituto Nacional de Antropología e Historia de Mexico, participa en esta convocatoria con el texto "CULTURA CUBANA Y MEDIOS DE COMUNICACIÓN EN MÉXICO, 1920-1950”. En este artículo, destaca el profesor Pérez Montfort, estudiamos las huellas que la cultura popular cubana, durante la primera mitad del siglo XX, imprimió en la cultura popular mexicana, a través de los medios de comunicación; estamos hablando de la prensa, el radio y el cine, en particular. Este proceso muestra cómo las características de los estereotipos culturales identificados con lo 
cubano, generaron un producto de exportación que los medios mexicanos supieron aprovechar de maneras muy creativas. Esto puede rastrearse, en particular, en la música y el cine producidos en los años treinta y cuarenta. Los teatros, salones de baile y cabarets fueron otros espacios en los que este imaginario cobró forma. Detrás de esto hubo una nutrida migración de artistas cubanos que contribuyeron a la manufactura de una vertiente de la cultura popular mexicana asociada a lo tropical. La relación personal entre estos cubanos y cubanas, y sus comparsas mexicanos, así como los vínculos que muchos de los oriundos de la Gran Antilla tuvieron con empresarios del espectáculo y productores y directores de cine en México fueron piezas claves en esta historia.

El historiador de la Universidad de Cartagena y productor de la emisora universitaria UdeC Radio, Luis Eduardo Mestra Narváez, contextualiza un importante período socio-cultural del país y la ciudad en el texto "LA PRENSA: ESPACIO DEL PODER Y FORMACIÓN DE OPINIÓN PÚBLICA EN CARTAGENA, 1920-1940". Las décadas de los años 20 al 40 del siglo pasado se constituyeron en un período de amplio desarrollo para las principales ciudades del país, coyuntura que permitió en Cartagena el fortalecimiento de aspectos como la industrialización, la educación y el acceso masivo a la información. No obstante, dicho período estuvo marcado por la legitimación de modelos de desarrollo y proyección de tribunas informativas pero bajo un tinte tradicional de camaradería política partidista. En este contexto, la formación de la opinión pública en la ciudad se vio condicionada por el bajo nivel de escolaridad de la mayoría de la población y el permanente enfrentamiento de los partidos hegemónicos en el país, manifiesto en los principales periódicos de la época, los cuales hacían parte de toda una infraestructura social donde la intervención de la clase burguesa local y sus ideologías políticas buscaban, en lo posible, atraer la balanza a su favor.

En la presente edición de PALOBRA, "palabra que obra" se reseñan tres libros. La primera a cargo de los estudiantes Stephanie Beltrán Paredes, Raúl Cera Ochoa, Estudiantes del programa de Historia de la Universidad de Cartagena, pertenecientes al grupo de investigación Masculinidades y Feminidades: "Debates sobre ciudadanía y políticas raciales en las Américas Negras", Bogotá, Facultad de Ciencias Humanas, Universidad del Valle, 2010, 992 p. Cuya autoría es compartida por los profesores Claudia Mosquera Rosero Labbé, Agustín Laó Montes, César Rodríguez Garavito (editores y coautores).

Palobra No. 12. Agosto de 2010 - Julio de 2011 
Este texto incluye artículos que responden a temáticas que se articulan en torno al problema de la raza como concepto y al racismo como práctica y a su relación con la ciudadanía, la inclusión, la exclusión, la colonialidad y sus formas de violencia; temas, sin duda relevantes para poder interpretar la dinámica social en Latinoamérica, son planteados y analizados desde diversas perspectivas. En síntesis, el libro de los profesores Claudia Mosquera Rosero Labbé, Agustín Laó Montes y César Rodríguez Garavito, además de estar generosamente acompañado por un amplio conjunto de fotografías sobre el desplazamiento forzado, arroja luces para las discusiones que habrán de continuar a propósito del concepto de raza y su influencia en los países de América Latina.

El segundo texto "Regionalización y Movimiento de Mujeres: Procesos en el Caribe Colombiano", escrito por la investigadora Yusmidia Solano Suarez, Instituto de Estudios del Caribe. Universidad Nacional de Colombia. Sede Caribe. San Andrés. 2006. Con 287 páginas es reseñado por los estudiantes Carlos Mario Castro y Nathaly Ortega Polanco de los programas de historia Y Trabajo Social e integrantes del semillero Estudio de familias Masculinidades y feminidades. Polanco.

El texto compila los principales procesos de regionalización en el Caribe Colombiano y como dentro de estos, las mujeres crearon movimientos políticos en donde fueron principales contribuyentes de aspectos sociales a la vida cotidiana, analizando los principales cambios que generaron las dinámicas de regionalización, que mas adelante crearán un ambiente propicio para la participación e interés en las mujeres que hacen parte de la región propiciando un cambio en su situación. Se destaca el papel de los discursos e identidad de mujeres y hombres, el cual esta expresada en determinadas formas de ser y actuar, lo cual ha sido un gran aporte de las culturas africanas, aborígenes y española.

El tercer texto reseñado "En la Onda de la radio", de la profesora de la Universidad de Cartagena Berta Lucia Arnedo, la realiza Winston Morales. "Ella, al igual que millones de Homo Consumus (llámese hombre), le debe su vida a la radio, Todo lo que es, o casi todo, nace y termina en el medio"... resalta Morales; y ante esas deudas colosales, Bertha Lucía siente la necesidad de pagarle con creces a uno de los medios calientes que perviven pese a sus más de 100 años de existencia. De allí viene su libro, de esa retribución coherente con lo que es su oficio como docente universitaria. Bertha, y de eso pueden dar 
fe todos sus condiscípulos, ama el medio, ama la radio, ama los micrófonos. Para quienes amamos la radio, como sé que la ama ella, el medio no es sólo el mensaje: la radio es incluso lo inexpresado, el silencio, lo que se calla. Pero es también la vida, atmósfera, sustancia, aceite para las ruedas por las que circula el mundo.

En notas de aquí... y Notas de allá se presentan eventos en los que tienen participación los integrantes de los grupos de investigación de La Facultad de Ciencias Sociales Y Educación. Además incluimos información sobre los jóvenes investigadores de los grupos quienes han asumido un importante liderazgo en esta edición.

El comité editorial de la presente edición, agradece a la comunidad académica nacional e internacional de las ciencias sociales, humanas y educativas, que se hizo presente en este número, fortaleciendo las condiciones de exigencia en los indicadores internacionales de revistas indexadas. Comprometido con la calidad editorial, pone a discusión y crítica este volumen a nuestros lectores y esperamos seguir alimentando el trabajo académico e investigativo, animándonos a superar cada día, los retos y compromisos que hemos asumido.

Dora Piñeres De la Ossa, Editora. 